\title{
ON THE SUM OF DIGITS OF PRIMES IN IMAGINARY QUADRATIC FIELDS
}

\author{
By \\ Ryuji Kaneiwa and Iekata SHIoKawa
}

1. Introduction. Let $r \geqq 2$ be a fixed integer. Any positive integer $n$ can be uniquely written in the form

$$
n=\sum_{j=1}^{k} a_{j} r^{k-j}=a_{1} a_{2} \cdots a_{k},
$$

where each $a_{j}$ is one of $0,1, \cdots, r-1$ and

$$
k=k(n)=\left[\frac{\log n}{\log r}\right]+1,
$$

where $[u]$ is the integral part of the real number $u$. We put

$$
s(n)=\sum_{j=1}^{k} a_{j} .
$$

I. Kátai [1] proved, assuming the validity of density hypothesis for the Riemann zeta function, that

$$
\sum_{p \leq x} s(p)=\frac{r-1}{2 \log r} x+O\left(\frac{x}{(\log \log x)^{1 / 3}}\right),
$$

where in the sum $p$ runs through the prime numbers. The second-named author [6] proved, without any hypothesis, the result of Kátai with an improved remainder term

$$
O\left(x\left(\frac{\log \log x}{\log x}\right)^{1 / 2}\right)
$$

His method is to appeal to a simple combinatorial inequality (see Lemma in $\S 4$ ), and the deepest result on which he depends is the prime number theorem in a weak form

$$
\sum_{p \leqq x} 1=\frac{x}{\log x}+O\left(\frac{x}{(\log x)^{2}}\right)
$$

E. Heppner [2] independently proved a more general result by making use of a Chebyshev's inequality to the sum of independent random variables (cf. [5] p. 387, Theorem 2): Let $B$ be a set of positive integers such that

Received May 29, 1978. Revised January 12, 1980. 


$$
\log \frac{x}{B(x)}=o(\log x)
$$

where

$$
B(x)=\sum_{\substack{n \leq x \\ n \in B}} 1
$$

Then

$$
\sum_{\substack{n \leq x \\ n \in B}} s(n)=\frac{r-1}{2} \frac{\log x}{\log r} B(x)\left(1+O\left(\left(\frac{\log \log x+\log \frac{x}{B(x)}}{\log x}\right)^{1 / 2}\right) .\right.
$$

This together with (4) implies (3).

In the present paper we shall show that the estimate (3) is also valid. in some sense, for primes in each imaginary quadratic field $\boldsymbol{Q}(\sqrt{-m})$, where $m$ is any positive square free integer.

2. Representation of integers in $Q(\sqrt{-m})$ in the scale of $r$. Let $\mathfrak{o}$ be the ring of all integers in $\boldsymbol{Q}(\sqrt{-m})$. Any $\alpha \in \mathfrak{p}$ can be expressed in a unique way as

where

$$
\alpha=a+b \omega \quad(a, b \in \boldsymbol{Z}),
$$

$$
\omega=\left\{\begin{array}{lll}
\sqrt{-m} & \text { if }-m \equiv 2,3(\bmod 4), \\
\frac{1+\sqrt{-m}}{2} & \text { if }-m \equiv 1 \quad(\bmod 4),
\end{array}\right.
$$

and $Z$ denotes as usual the set of all rational integers. So by means of the expessions

$$
|a|=a_{1} a_{2} \cdots a_{k(|a|),}|b|=b_{1} b_{2} \cdots b_{k(|b|)}
$$

given by (1), we can define coordinatewisely the representation of $\alpha \in \mathfrak{D}$ in the scale of $r$; i. e.

$$
\alpha=\sum_{j=1}^{k} \alpha_{j} r^{k-j}=\alpha_{1} \alpha_{2} \cdots \alpha_{k}
$$

where

$$
\begin{aligned}
& k=k(\alpha)=\max \{k(|a|), k(|b|)\}, \quad k(0)=1, \\
& \alpha_{j}=\operatorname{sgn}(a) a_{j}+\operatorname{sgn}(b) b_{j} \omega,
\end{aligned}
$$

and $\operatorname{sgn}(c)=c /|c|$ if $c \neq 0,=0$ otherwise. We define

$$
s(\alpha)=\sum_{j=1}^{k} \alpha_{j} .
$$

We write

$$
\mathcal{A}_{1}=\{a+b \omega \mid a, b \in Z ; a \geqq 0, b \geqq 0\},
$$


On the sum of digits of primes in imaginary quadratic fields

$$
\begin{aligned}
& \mathcal{A}_{2}=\left\{-a+b \omega \mid a+b \omega \in \mathcal{A}_{1}\right\}, \\
& \mathcal{A}_{3}=\left\{-a-b \omega \mid a+b \omega \in \mathcal{A}_{1}\right\}, \\
& \mathcal{A}_{4}=\left\{a-b \omega \mid a+b \omega \in \mathcal{A}_{1}\right\},
\end{aligned}
$$

so that $\mathfrak{D}=\mathscr{A}_{1} \cup \mathcal{A}_{2} \cup \mathcal{A}_{3} \cup \mathcal{A}_{4}$. We denote by $\mathscr{B}_{i}$ the set of all 'digits' $\alpha_{j}$ needed for the expressions (5) of all $\alpha \in \mathcal{A}_{j}$. Then

$$
\begin{aligned}
& \mathscr{B}_{1}=\{c+d \omega \mid c, d=0,1, \cdots, r-1\}, \\
& \mathscr{B}_{2}=\left\{-c+d \omega \mid c+d \omega \in \mathscr{B}_{1}\right\}, \\
& \mathscr{B}_{3}=\left\{-c-d \omega \mid c+d \omega \in \mathscr{B}_{1}\right\}, \\
& \mathscr{B}_{4}=\left\{c-d \omega \mid c+d \omega \in \mathscr{B}_{1}\right\},
\end{aligned}
$$

and card $\mathscr{B}_{i}=r^{2}(1 \leqq i \leqq 4)$. So we may say that the $r$-adic expression (5) of $\alpha \in \mathfrak{D}$ is a kind of representation in the scale of $r^{2}$. For any fixed $\beta \in \mathscr{B}_{i}$ we denote by $F(\alpha, \beta)$ the number of $\beta$ appearing in the expression (5) of an integer $\alpha \in \mathcal{A}_{1}$. By definition

$$
s(\alpha)=\sum_{\beta \in \mathfrak{B}_{i}} \beta F(\alpha, \beta) \quad\left(\alpha \in \mathcal{A}_{i}\right)
$$

and

$$
\begin{aligned}
& F(a+b \omega, c+d \omega)=F(-a+b \omega,-c+d \omega) \\
& \quad=F(-a-b \omega,-c-d \omega)=F(a-b \omega, c-d \omega) \quad(a, b \in \boldsymbol{Z}) .
\end{aligned}
$$

The norm of $\alpha=a+b \omega \in \mathfrak{D}$ is a rational integer

$$
N(\alpha)=\left\{\begin{array}{lll}
a^{2}+m b^{2} & \text { if }-m \equiv 2,3(\bmod 4) \\
a^{2}+a b+\frac{m+1}{4} b^{2} & \text { if }-m \equiv 1 \quad(\bmod 4)
\end{array}\right.
$$

so that for $\alpha \neq 0$

$$
\left|k(\alpha)-\frac{\log N(\alpha)}{2 \log r}\right| \leqq c_{1},
$$

where $c_{1}$ is a constant depending only on $m$, since by definition

$$
\left|k(a)-\frac{\max (\log |a|, \log |b|)}{\log r}\right| \leqq 1
$$

(we mean that $\max (\log 0, x)=x$ ) and

$$
\begin{aligned}
& |2 \max (\log |a|, \log |b|)-\log N(\alpha)| \\
& \leqq\left\{\begin{array}{l}
\log (1+m) \quad \text { if }-m \equiv 2,3(\bmod 4), \\
\log \left(2+\frac{m+1}{4}\right) \text { if }-m \equiv 1 \quad(\bmod 4) .
\end{array}\right.
\end{aligned}
$$


3. A prime number theorem (A. Mitsui [3], [4]). An integer $\alpha \in \mathfrak{D}$ is said to be prime if $(\alpha)$ is an prime ideal in $\boldsymbol{Q}(\sqrt{-m})$. Let $\theta_{1}, \theta_{2}$ be two real numbers such that $0 \leqq \theta_{1}<\theta_{2} \leqq 2 \pi$. Then

$$
\begin{aligned}
& \sum_{\substack{\alpha: \text { prime } \\
N(x) \leq x \\
\theta_{1} \leq \arg \alpha \leq \theta_{2}}} 1=\frac{\left(\theta_{2}-\theta_{1}\right) w}{2 \pi h} \int_{2}^{x} \frac{d t}{\log t} \\
& \quad+O\left(x \exp \left(-c_{2}(\log x)^{3 / 5}(\log \log x)^{-1 / 5}\right)\right),
\end{aligned}
$$

where $h$ is the class number of $\boldsymbol{Q}(\sqrt{-m})$ and

$$
w=\left\{\begin{array}{lll}
4 & \text { if } & m=1, \\
6 & \text { if } & m=3, \\
2 & \text { otherwise }
\end{array}\right.
$$

We note that a weaker estimate $O\left(x /(\log x)^{2}\right)$ is sufficient for the proof of our theorem.

4. A combinatorial lemma (I. Shiokawa [6]). Let $\beta_{1}, \cdots, \beta_{g}$ be given $g$ symbols and let $A^{j}$ be the set of all sequences of these symbols of length $j \geqq 1$. Denote by $F_{j}(\alpha, \beta)$ the number of any fixed symbol $\beta$ appearing in a sequence $\alpha \in A^{j}$. Then for any $\varepsilon$ with $0<\varepsilon<1 / 2$ there exist a positive integer $j_{0}$ independent of $\varepsilon$ such that the number of sequences $\alpha \in A^{j}$ satisfying

$$
\left|F_{j}(\alpha, \beta)-\frac{j}{g}\right|>j^{1 / 2+\varepsilon}
$$

is less that $j g^{j} \exp \left(-c_{3} j^{2 \varepsilon}\right)$ for all $j \geqq j_{0}$, where $c_{3}$ is an absolute constant.

5. Theorem. Let $\varphi_{1}=0, \varphi_{5}=2 \pi, \varphi_{2}=\arg \omega, \varphi_{3}=\pi$, and $\varphi_{4}=\varphi_{2}+\pi$. Then for any $\theta_{1}, \theta_{2}$ satisfying $\varphi_{j} \leqq \theta_{1}<\theta_{2} \leqq \varphi_{j+1}$ for some $j$ we have

$$
\begin{gathered}
\sum_{\substack{\alpha: \operatorname{prime} \\
N \leq \alpha)=\\
\theta_{1} \leqq \arg \alpha \leq \theta_{2}}} s(\alpha)=\frac{\left(\theta_{2}-\theta_{1}\right) w}{2 \pi h} \frac{(r-1)}{4 \log r} \lambda_{j} x \\
+O\left(x\left(\frac{\log \log x}{\log x}\right)^{1 / 2}\right),
\end{gathered}
$$

where

$$
\lambda_{j}=\left\{\begin{array}{rll}
1+\omega & \text { if } & j=1, \\
-1+\omega & \text { if } & j=2, \\
-1-\omega & \text { if } & j=3, \\
1-\omega & \text { if } & j=4
\end{array}\right.
$$

and the $O$-constant depends at most on $r$ and $m$. 
6. Proof of Theorem. By (7) and (8) we may assume $j=1$. We define for $\alpha \in \mathcal{A}_{1}$ and $\beta \in \mathscr{B}_{1}$

$$
D(\alpha, \beta)=\left|F(\alpha, \beta)-\frac{k(\alpha)}{r^{2}}\right|
$$

Put for brevity

$$
\mathcal{C}(x)=\left\{\alpha \in \mathfrak{D} \mid \alpha: \text { prime, } N(\alpha) \leqq x, \theta_{1} \leqq \arg \alpha \leqq \theta_{2}\right\} .
$$

Then by (7) and (12)

$$
\begin{aligned}
\sum_{\alpha \in \mathcal{C}^{\prime}(x)} s(\alpha) & =\sum_{\beta \in \mathscr{S}_{1}} \beta \sum_{\alpha \in \mathcal{L}^{\prime}(x)} F(\alpha, \beta) \\
& =\frac{r-1}{2} \lambda_{1} \sum_{\alpha \in \mathcal{C}^{\prime}(x)} k(\alpha)+O\left(\sum_{\beta \in \mathcal{B}_{1}} \sum_{\alpha \in C^{\prime}(x)} D(\alpha, \beta)\right) .
\end{aligned}
$$

By (9) and (10) we have

$$
\sum_{\alpha \in \mathcal{C}^{\prime}(x)} k(\alpha)=\frac{\left(\theta_{2}-\theta_{1}\right) w}{2 \pi h} \frac{x}{2 \log r}+O\left(\frac{x}{\log x}\right) .
$$

Put $D(\alpha)=D\left(\alpha, \beta_{0}\right)$, where $\beta_{0}$ is any fixed integer in $\mathscr{B}_{1}$. We have from (9), $(10)$, and (12)

$$
\begin{aligned}
& \sum_{x \in \mathcal{C}(\alpha)} D(\alpha) \leqq \sum_{\alpha \in \mathcal{C}(x)} k(\alpha)^{1 / 2+\varepsilon}+\sum_{\substack{\alpha \in \mathcal{C}(x) / 2+\varepsilon \\
D(\alpha)>k(\alpha) 1 / 2+\varepsilon}} D(\alpha) \\
& =O\left(\sum_{\alpha \in \mathcal{C}(x)}(\log N(\alpha))^{1 / 2+\varepsilon}\right)=O\left(\sum_{\substack{\alpha \in \in \mathcal{A}_{1} \\
N(\alpha) \leq x \\
D(\alpha)>k(\alpha) 1 / 2+\varepsilon}} D(\alpha)\right. \\
& \left.=O(x \log x)^{\varepsilon-1 / 2}\right)+O\left(\log x \sum_{\substack{\alpha \in \mathcal{H}_{1} \\
N(\alpha) \leq x \\
D(\alpha)>k(\alpha) 1 / 2+\varepsilon}} 1\right) .
\end{aligned}
$$

Besides, using (9),

$$
\sum_{\substack{\alpha \in \mathcal{A}_{1} \\
N(\alpha) \leq x \\
D(\alpha)>k(\alpha) 1 / 2+\varepsilon}} 1 \leqq \sum_{j \leqq l(x)} \sum_{\begin{array}{c}
\alpha \in \mathcal{A l}_{1} \\
k(\alpha)=j \\
D(\alpha)>j 1 / 2+\varepsilon
\end{array}} 1
$$

where

$$
l(x)=\frac{\log x}{2 \log r}+c_{1} .
$$

Applying now the lemma in $\S 4$ with $g=r^{2}$ and $A^{1}=\mathscr{B}_{1}$, we get

$$
\sum_{\substack{\alpha \in \mathcal{A}_{1} \\ k(\alpha)=j \\ D(\alpha)>j^{1 / 2+\varepsilon}}} 1<j r^{2 j} \exp \left(-c_{3} j^{2 \varepsilon}\right)
$$

for all $j \geqq j_{0}$, which leads to

$$
\sum_{\substack{\alpha \in \in \mathcal{A}_{1} \\ N(\alpha) \leq x^{\prime} \\ D(\alpha)>k(\alpha) 1 / 2+\varepsilon}} 1=O(1)+\sum_{j_{0}<j \leq l(x)} j r^{2 j} \exp \left(-c_{3} j^{2 \varepsilon}\right)
$$




$$
\begin{aligned}
& =O(1)+\sum_{j_{0}<j \leq l(x) / 2}+\sum_{l(x) / 2<j \leq l(x)} \\
& =O\left(x(\log x)^{2} \exp \left(-\frac{c_{3}}{4}\left(\frac{\log x}{\log r}\right)^{2 \varepsilon}\right)\right) .
\end{aligned}
$$

where the $O$-constant is uniform in $\varepsilon$.

If we take a constant $c_{4}=c_{4}(r)$ large enough and choose $\varepsilon=\varepsilon(x, r)$ with $0<\varepsilon<1 / 2$ in such a way that

$$
(\log x)^{2 \varepsilon}=c_{4} \log \log x
$$

we obtain from (15) and (16)

$$
\sum_{\alpha \in \mathcal{C}(x)} D\left(\alpha, \beta_{0}\right)=O\left(x\left(\frac{\log \log x}{\log x}\right)^{1 / 2}\right) .
$$

This together with (13) and (14) yealds the theorem.

\title{
References
}

[1] Kátai, I., On the sum of digits of prime numbers. Ann. Univ. Sci. Budapest Roland Eötvös nom. Sect. Math., 10 (1967), 89-93.

[2] Heppner, E., Über die summe der Ziffern naturlicher Zahlen. Ann. Univ. Sci. Budapest Roland Eötvos nom. Sect. Math., 19 (1976), 41-43.

[3] Mitsui, T., Generalized prime number theorem. Japanese J. Math., 26 (1956) , 1-42.

[4] Mitsui, T., Research Institute for Mathematical Sciences Kyoto Univ., "Kokyuroku" 294 (1977), in Japanese.

[5] Rényi, A., Probability Theorey, North-Holland, Amsterdam-London, 1970.

[6] Shiokawa, I., On the sum of digits of prime numbers. Proc. Japan Acad., 50, no. 8 (1974), 551-554.

\author{
Ryuji Kaneiwa \\ Institute of Liberal Arts \\ Otaru University of Commerce \\ Otaru, Hokkaido \\ 047 Japan \\ Iekata Shiokawa \\ Department of Mathematics \\ Keio University \\ Yokohama \\ 223 Japan
}

